\title{
Isochronicity and Commutation of Polynomial Vector Fields
}

\author{
E. P. Volokitin and V. V. Ivanov*
}

\begin{abstract}
We study a connection between the isochronicity of a center of a polynomial vector field and the existence of a polynomial commuting system. We demonstrate an isochronous system of degree 4 which does not commute with any polynomial system. We prove that among the Newton polynomial systems only the Lienard and Abel systems may commute with transversal polynomial fields. We give a full and constructive description of centralizers of the Abel polynomial systems. We give new examples of isochronous systems.
\end{abstract}

A center of a planar dynamical system is said to be isochronous if all cycles near it have the same period. The isochronicity problem has a long history [1]. After two or three decades of oblivion, the interest in this problem has been revived partially due to proliferation of powerful methods of computerized research. At this juncture, most attention is paid to polynomial vector fields (see, for instance, [2, 3] and the bibliography therein). The characteristic feature of the present-day activity is the fact that some long forgotten results are rediscovered and at the same time new nontrivial examples of isochronous systems appear, giving rise to fresh and ingenious ideas.

\section{A Dynamical Meaning of the Cauchy-Riemann Conditions}

One of these ideas leans upon a remarkable observation by Villarini [4] which allowed him to gave an elegant proof to a well-known theorem by Lukashevich [1, 可. Lukashevich's theorem asserts that each center of a system

$$
\begin{aligned}
& \dot{x}=p(x, y), \\
& \dot{y}=q(x, y)
\end{aligned}
$$

whose right-hand sides satisfy the Cauchy-Riemann conditions

$$
p_{x}=q_{y}, \quad p_{y}=-q_{x}
$$

*The research was supported by the Russian Foundation for Basic Research (Grants 99-01-00574, 9901-00575). 
is isochronous.

From the viewpoint of dynamics, these conditions mean that system (1) commutes with the orthogonal system

$$
\begin{aligned}
\dot{x} & =q(x, y), \\
\dot{y} & =-p(x, y) .
\end{aligned}
$$

Indeed, the commutator, or Lie bracket [6], of the vector fields $(p, q)$ and $(q,-p)$ is the field with the components

$$
\begin{aligned}
& q\left(p_{x}-q_{y}\right)-p\left(p_{y}+q_{x}\right), \\
& p\left(p_{x}-q_{y}\right)+q\left(p_{y}+q_{x}\right)
\end{aligned}
$$

whose vanishing at a noncritical point with $p^{2}+q^{2} \neq 0$ is equivalent to the fulfillment of the Cauchy-Riemann conditions.

Thus, if a point, moving according to equations (1), returns to the initial position at time $T$ and the orthogonal flow (2) afterwards carries it to a nearby orbit of (四), then the fact that the flows commute implies that the motion of the point along the latter by time $T$ also terminates at the same place where it starts.

This argument proves Lukashevich's theorem and also allows us to make some interesting conclusions about the properties of the system under consideration. For example, the orbits of such a system, lying nearby a cycle, are necessarily closed, so that there are no limit cycles. Moreover, if an analytical system is defined over a simply connected domain then each cycle of the system surrounds a unique singular point, which is an isochronous center, and lies in the basin of this center together with the region that it bounds.

We now consider an arbitrary system (1) and suppose that it commutes with another system

$$
\begin{aligned}
& \dot{x}=r(x, y), \\
& \dot{y}=s(x, y) .
\end{aligned}
$$

In the language of vector fields, this amounts to validity of the equalities

$$
\begin{aligned}
& r p_{x}+s p_{y}-p r_{x}-q r_{y}=0 \\
& r q_{x}+s q_{y}-p s_{x}-q s_{y}=0
\end{aligned}
$$

Then the above argument clearly remains valid in each domain where systems (1) and (3) are transversal. In particular, if such domain surrounds a center of system (11) then the center is isochronous. It was Sabatini [7] who noticed this fact. He also proved the converse assertion, namely: in a punctured neighborhood of an isochronous center there always exists a system transversal to the original system and commuting with it; moreover, this system has the same smoothness as the original system. This gives a new tool for proving isochronicity of various systems in a uniform way.

It is worth noting that conditions (4) amount merely to local commutation of the flows $U_{\tau}$ and $V_{\sigma}$ corresponding to systems (11) and (3), even if the corresponding vector fields are defined on the whole plane. Surely, sometimes we can extend equality between the values $V_{\sigma} U_{\tau}$ and $U_{\tau} V_{\sigma}$ at a given point to rather large $\tau$ and $\sigma$, for example, when the products $V_{\sigma^{\prime}} U_{\tau^{\prime}}$ and $U_{\tau^{\prime}} V_{\sigma^{\prime}}$ make sense at this point for all pairs $\sigma^{\prime}, \tau^{\prime}$ in the rectangle $0 \leq \sigma^{\prime} \leq \sigma$, $0 \leq \tau^{\prime} \leq \tau$. However, we cannot guarantee the equality $V_{\sigma} U_{\tau}=U_{\tau} V_{\sigma}$ on the only reason that 
both sides of the equality are defined at the point in question. Neglect of this circumstance might lead to erroneous conclusions. For example, by an argument similar to that above, one might conclude that two isochronous centers whose basins are joined by an orbit of a commuting system have the same period. However, this is not always the case. We exhibit one example.

A system $\dot{z}=f(z)$ on the plane, with $f(z)$ a holomorphic function of a complex variable $z$, has a center at a point $z_{0}$ if and only if $f\left(z_{0}\right)=0$ and the derivative $f^{\prime}\left(z_{0}\right)$ is a nonzero purely imaginary number. This result is also due to Lukashevich [5], but essentially the same result was recently reproved in [8]. Thus, if, say, $f(z)=i z\left(1-z^{2}\right)$ then our system has three centers. They all lie on the real axis, separating it into four parts each of which, as we easily see, serves as an orbit of the orthogonal system $\dot{z}=-i f(z)$. Two of them join the point $(0,0)$ to the points $(-1,0)$ and $(1,0)$. Moreover, the extreme centers have period $\pi$, whereas the period of the middle center equals $2 \pi$.

We now study the mechanism of this phenomenon in more detail. The essential elements of the phase portrait of the system under consideration are displayed in Fig. 1. Two separatrices infinite to both sides separate the plane into three domains. These domains are entirely covered by cycles. The whole picture is symmetric about the coordinate axes.

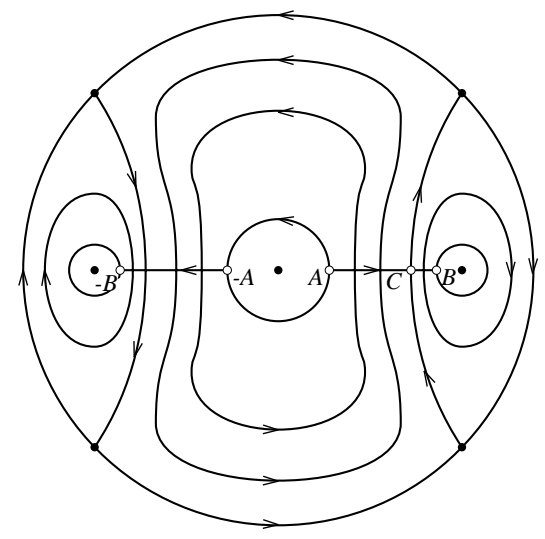

Fig. 1

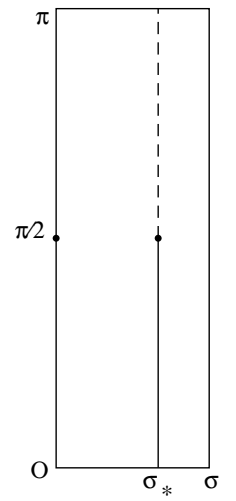

Fig. 2

In the orthogonal system, consider the motion starting at $t=0$ at the point $A$ indicated in the picture and finishing at some time $t=\sigma$ at the point $B$ lying at the intersection of one of the orbits around the right center with the abscissa axis. If we use the former notations $U$ and $V$ for the original and orthogonal flows and take symmetry of the system and the above-indicated values of periods into account, then we come to the following expressive relations:

$$
U_{\pi} V_{\sigma}(A)=U_{\pi}(B)=B, \quad V_{\sigma} U_{\pi}(A)=V_{\sigma}(-A)=-B .
$$

Certainly, the culprits are the separatrices separating the basins of the centers: their blame consists in their going to infinity too fast, so that they create obstacles to extending commutation of the flows to the needed range. Indeed, we can easily calculate that these separatrices represent two branches of the hyperbola $2 x^{2}-2 y^{2}=1$ and the motion along 
them is described by the explicit formulas

$$
x= \pm \frac{\cos (t / 2)}{\sqrt{2 \cos t}}, \quad y= \pm \frac{\sin (t / 2)}{\sqrt{2 \cos t}} .
$$

The duration of traversing each of these orbits embeds in the interval $-\pi / 2<t<+\pi / 2$ of length $\pi$. Thus, if $\sigma_{*}$ is the moment when the point $C=V_{\sigma_{*}}(A)$ occurs on the right separatrix then the orbit $U_{t}(C)$ terminates by time $t=\pi / 2$. Now, disregarding the fact that the other orbits of the flow $U$ which start at the points of the segment $A B$ live for the infinite time, there is no "commuting" rectangle which could equate the values of $V_{\sigma} U_{\pi}$ and $U_{\pi} V_{\sigma}$ at $A$ (Fig. 2).

Seizing an opportunity, we indicate one more curious property of the periods of polynomial analytical systems.

Theorem 1 If a polynomial system of degree $n \geq 2$ satisfies the Cauchy-Riemann conditions and has $n$ singular points such that $n-1$ of them are centers, then the remaining point must be a center too. Furthermore, among all singular points there necessarily exist those around which the rotation is clockwise as well as those around which the rotation is counterclockwise. Finally, the sums of periods for the various types of centers are the same.

Proof. If among the roots $z_{1}, \ldots, z_{n}$ of a polynomial $f(z)$ of degree $n \geq 2$ there are $n-1$ simple roots then it is clear that the remaining root is simple as well. Furthermore, the following remarkable identity is easy to verify:

$$
\frac{1}{f^{\prime}\left(z_{1}\right)}+\ldots+\frac{1}{f^{\prime}\left(z_{n}\right)}=0 .
$$

If all points $z_{k}$ with $k=1, \ldots, n-1$ are centers of the system $\dot{z}=f(z)$ then, as was mentioned above, the numbers $f^{\prime}\left(z_{k}\right)$ are purely imaginary. But then the preceding identity implies that the derivative $f^{\prime}\left(z_{n}\right)$ must be purely imaginary as well. Since this derivative differs from zero, the point $z_{n}$ must be a center. It remains to observe that the absolute value of the real number $2 \pi i / f^{\prime}\left(z_{k}\right)$ equals the period of cycles around the (isochronous) center $z_{k}$ and the sign of this number determines the direction of the circuit.

\section{Sabatini's Problem and Devlin's Example}

We now discuss the application of the above approach to studying isochronous centers of polynomial dynamical systems. The following problem was stated in the already-quoted article [7]:

Problem. Is it true that a polynomial system, having an isochronous center, commutes with some transversal polynomial system?

For every quadratic system with an isochronous center, in [9] the author of this question constructed a commuting polynomial system whose linear part is orthogonal to the original linear system. Using Mathematica [10], we examined all isochronous polynomial systems 
described in the articles we know. It is surprising, but for the majority of them the answer to the above question turned out to be affirmative. We outline the results of our inspection.

Example 1. The article [2] contains a full list of all (up to homothety) cubic systems symmetric with respect to an isochronous center. It is as follows:

$$
\begin{array}{ll}
\dot{x}=-y-3 x^{2} y+y^{3}, & \dot{x}=-y+x^{2} y, \\
\dot{y}=x+x^{3}-3 x y^{2}, & \dot{y}=x+x y^{2}, \\
\dot{x}=-y+3 x^{2} y, & \dot{x}=-y-3 x^{2} y, \\
\dot{y}=x-2 x^{3}+9 x y^{2}, & \dot{y}=x+2 x^{3}-9 x y^{2} .
\end{array}
$$

Below we indicate the corresponding polynomial systems, obviously transversal to the original systems at least near zero, which commute with them as one can check by straightforward verification of identities (四):

$$
\begin{aligned}
& \dot{x}=x+x^{3}-3 x y^{2}, \quad \dot{x}=x-x^{3} \text {, } \\
& \dot{y}=y+3 x^{2} y-y^{3}, \quad \dot{y}=y-x^{2} y \text {, } \\
& \dot{x}=x-5 x^{3}+6 x^{5}, \quad \dot{x}=x+5 x^{3}+6 x^{5} \text {, } \\
& \dot{y}=y-9 x^{2} y+18 x^{4} y, \quad \dot{y}=y+9 x^{2} y+18 x^{4} y \text {. }
\end{aligned}
$$

Example 2. As is well known [2], among Kukles's systems we have exactly one system (up to a similarity transformation) with an isochronous center:

$$
\begin{aligned}
& \dot{x}=-y, \\
& \dot{y}=x+3 x y+x^{3} .
\end{aligned}
$$

For it, there exists a transversal commuting polynomial system:

$$
\begin{aligned}
& \dot{x}=x+x y+x^{3}, \\
& \dot{y}=y-x^{2}+y^{2}-x^{4} .
\end{aligned}
$$

As computer analysis shows, the above isochronous system remains unique even in the full class of Kukles's systems when the second equation is specified by an arbitrary polynomial of third degree. Some relevant remarks can be found, for instance, in [8].

It is curious to notice that each of the isochronous systems of the shape

$$
\begin{aligned}
& \dot{x}=-y+p_{2}(x, y), \\
& \dot{y}=x+q_{2}(x, y),
\end{aligned}
$$

where $p_{2}$ and $q_{2}$ are homogeneous quadratic polynomials written in the form they are presented in Table 1 of [2], in the variables $X=x, Y=y-p_{2}(x, y)$ coincides, up to a similarly transformation, with the above-written Kukles's system.

Truly ubiquitous, this system plays a magical role in the problem under study. We encounter it once more in our article, moreover, at a place where we did not expect to see it at all. 
Example 3. Studying the cubic Kolmogorov systems, the authors of [11 arrived at the following system with an isochronous center at the origin:

$$
\begin{aligned}
& \dot{x}=-y+2 x y-a x^{2} y, \\
& \dot{y}=x-x^{2}+y^{2}-a x y^{2} .
\end{aligned}
$$

This system commutes, for example, with the polynomial system

$$
\begin{aligned}
& \dot{x}=x-x^{2}+y^{2}-a x y^{2}, \\
& \dot{y}=y-2 x y-a y^{3} .
\end{aligned}
$$

It is noteworthy that, in all three examples of systems, their direction fields are symmetric about the abscissa axis, which makes it obvious that the origin is a center of each of them. Having constructed transversal commuting systems, we obtain one more proof for isochronicity of these centers.

Example 4. A new family of isochronous cubic systems was found in [3]. Slightly changing notations, we can write it as follows:

$$
\begin{aligned}
& \dot{x}=-y-2 x y+x^{2}(\alpha+\alpha x+\beta y), \\
& \dot{y}=x+x^{2}-y^{2}+x y(\alpha+\alpha x+\beta y) .
\end{aligned}
$$

For arbitrary real $\alpha$ and $\beta$, the origin is a center of the system, which is easy to check by using the first integrals indicated in [3]. Incidentally, everything happened just the other way about: the authors started with choosing an integral and then wrote a system for it. They studied the result by the Darboux linearization method and proved isochronicity of the center. Another proof of isochronicity appears on observing that the system in question commutes with the following:

$$
\begin{aligned}
& \dot{x}=x+x^{2}-y^{2}+x y(\alpha+\alpha x+\beta y), \\
& \dot{y}=y+2 x y+y^{2}(\alpha+\alpha x+\beta y) .
\end{aligned}
$$

As we can see, this example contains the previous: it suffices to take $\alpha=0$ and $\beta=-a$ and change the sign of the variables $x$ and $y$.

Example 5. A remarkable class of isochronous hamiltonian systems relates to areapreserving transformations [12]. Suppose that functions $u=u(x, y)$ and $v=v(x, y)$ satisfy the identity

$$
u_{x} v_{y}-u_{y} v_{x} \equiv 1
$$

In that event, if $u=v=0$, say, at $x=y=0$ then the origin is an isochronous center of the system

$$
\begin{aligned}
& \dot{x}=-u u_{y}-v v_{y}, \\
& \dot{y}=u u_{x}+v v_{x} .
\end{aligned}
$$

This is obvious, since in the coordinates $u, v$ the system takes a standard form of the harmonic oscillator: $\dot{u}=-v, \dot{v}=u$. However, for us it is important to indicate an explicit form of a commuting system which is polynomial in case $u$ and $v$ are polynomials:

$$
\begin{aligned}
& \dot{x}=u v_{y}-v u_{y}, \\
& \dot{y}=-u v_{x}+v u_{x} .
\end{aligned}
$$


The fact, that this system really commutes with and is transversal to the original system, can be verified by straightforward calculations, but it is simpler to observe that in the coordinates $u, v$ the system is written as the system $\dot{u}=u, \dot{v}=v$ corresponding to the canonical Euler field $(u, v)$ obviously orthogonal to the field $(-v, u)$ of the harmonic oscillator and commuting with it.

It is easy to construct as many isochronous hamiltonian systems as we wish if we observe that the Jacobian of the mapping $(u, v)$ is preserved under the replacement of $u$ by $u+\varphi(v)$ or $v$ by $v+\psi(u)$, where $\varphi$ and $\psi$ are arbitrary smooth functions of one variable.

We thus see that the hope of a positive solution to Sabatini's problem had certain grounds. However, the existence of a commuting system, even in a small neighborhood of a singular point, imposes strong requirements on the global behavior of the system as soon as we talk about the polynomial vector fields. It is quite possible that these requirements are met by all cubic isochronous systems, but they are already incompatible with the degree of freedom of systems of forth degree. One of the circumstances in support of what was said is reflected in the following simple theorem slightly developing the arguments of the beginning of the article.

Theorem 2 If two polynomial systems commute in some domain and are not collinear in at least one point of the plane then every center of each of the systems is isochronous.

Proof. The points where a polynomial of two variables vanishes either cover the whole plane or constitute a closed nowhere dense set. Therefore, first, our systems commute on all of the plane and, second, the basin of every center of each of them includes a cycle that the other flow, commuting with the given system, intersects somewhere transversally. But then all familiar arguments apply and demonstrate that all nearby cycles have the same period. In this case the period, depending analytically on the orbit, has a constant value on the whole basin.

Thus, the natural question arises: Can two centers of a polynomial system coexist if one of them is isochronous and the other is not? In view of the preceding theorem, a positive answer to this question would be simultaneously a negative answer to Sabatini's problem. We found an appropriate example in the article [13], wherein the system

$$
\begin{aligned}
\dot{x} & =-y-x^{4}+4 x^{2} y^{2}+y^{4}, \\
\dot{y} & =x-4 x^{3} y
\end{aligned}
$$

is indicated which has one isochronous and two nonisochronous centers.

When our article was in print, we received from Sabatini a large, still unpublished survey of isochronous centers [14] which contains many of the commuting polynomial systems we consider, as well as a whole series of new examples. Interestingly, this survey also points to Devlin's example [13], moreover, in connection with the same question. 


\section{Homogeneous Perturbations of the Harmonic Oscillator}

Besides the above, there are other reasons for which an isochronous polynomial system may fail to commute with any nonproportional polynomial system. Each of the following systems can serve as an example:

$$
\begin{aligned}
& \dot{x}=-y, \\
& \dot{y}=x+x^{2 m-1} y\left(x^{2}+y^{2}\right),
\end{aligned}
$$

where $m$ is an arbitrary natural number. Here we have only one singular point and therefore the preceding arguments fail.

We have found and investigated this system before we learned about Sabatini's problem. Now, when the question has received an answer, we might confine ourselves merely to mentioning our example. However, since polynomial vector fields of degree higher than tree are not studied practically and any information could be useful, in this section we conduct a small investigation of system (5) which is curious in many respects.

Theorem 3 The origin is an isochronous center of system (5).

Proof. Symmetry of the direction field of (ref5) about the ordinate axis implies that the origin is a center of the system. We write the system in polar coordinates:

$$
\dot{\varrho}=\varrho^{2 m+2} \cos ^{2 m-1} \vartheta \sin ^{2} \vartheta, \quad \dot{\vartheta}=1+\varrho^{2 m+1} \cos ^{2 m} \vartheta \sin \vartheta
$$

Differentiating these equations with respect to $t$, we can easily verify that the function

$$
t-\vartheta-\frac{\varrho^{2 m+1} \cos ^{2 m+1} \vartheta}{2 m+1}
$$

remains constant along each orbit. If the orbit is closed and, in consequence, surrounds the origin, then during one circuit the angle $\vartheta$ receives the increment $2 \pi$ and the fractional summand of the above expression returns to the initial value. Therefore, the duration of the circuit equals $2 \pi$.

Theorem 4 The polynomial systems able to commute with system (5) may differ from (5) only by a constant factor.

Proof. Suppose that the field $(p, q)$ of system (5) commutes with a polynomial vector field $(r, s)$. When $p=-y$, as we have in our case, the first expression in (4) takes the shape

$$
s=y r_{x}-q r_{y} .
$$

Now, if we rewrite the second relation in (4) by using (6) and next compare summands from the viewpoint of their degree in $y$, not forgetting that the degree of the polynomial $q$ is exactly three, then we find that the degree $r$ cannot exceed one, so that

$$
r=r_{0}(x)+r_{1}(x) y
$$


The further, rather elementary examination leads to the equalities $r_{0}=0$ and $r_{1}=-c$, where $c$ is a constant. Hence, $r=-c y$ and so $s=c q$. Thus, the field $(r, s)$ is collinear to the field $(p, q)$ with a constant coefficient of proportionality.

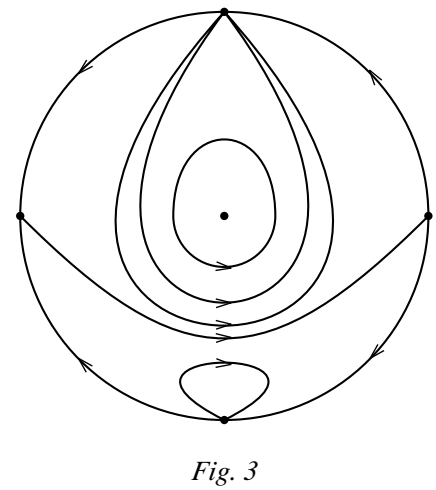

In Fig. 3 we depict the scheme of the phase portrait of system (5) which is obtained by the classical methods of the qualitative theory of planar dynamical systems. The above example of an isochronous system resulted from studying the one-dimensional nonconservative Newton equations with homogeneous nonlinearities added to the equation of the standard harmonic oscillator. On the phase plane, these equations are expressed by the system

$$
\begin{aligned}
& \dot{x}=-y, \\
& \dot{y}=x+Q_{n}(x, y),
\end{aligned}
$$

where $Q_{n}(x, y)$ is a homogeneous polynomial of degree $n$. Examining the known full lists [1, 2] of isochronous systems involving only quadratic or only cubic nonlinearities, we can observe that among them there are no Newton systems of the form (7) with $n=2$ and $n=3$. Inspecting the necessary conditions for isochronicity of a center by means of Mathematica, we verified that, up to homothety, there is exactly one isochronous system for $n=4$ : it is system (5) with the exponent $m=1$, and if $n=5$ then the origin never can be an isochronous center of system (7). By this we once more confirmed the results that were obtained thirty years ago in [15.

In the more difficult case of $n=6$, we succeeded only in examining systems that are symmetric about one of the coordinate axes. We found out that, with the previous stipulations, the only system among them which has an isochronous center at zero is system (5) with $m=2$. Finally, if $n=7$ then system (7) cannot have an isochronous center at zero.

The above consideration and some other reasons allow us to pose the following two questions.

Problem 1. Is it true that a system of the form (7) with nonlinearities of degree higher than two has a center at the origin if and only if its direction field is symmetric about one of the coordinate axes?

Problem 2. Is it true that the origin is an isochronous center of system (7) only if the system has an even degree and is reduced to system (5) by a similarity transformation?

Surely, in near future the progress in computers will advance the study of the class of systems in question far beyond the point we have reached. However, in this way we could 
hope to receive only a negative answer to the posed questions. If the answers are affirmative then the attempts at their justification will probably lead to new profound ideas. These questions are also closely connected with the other problems to be dealt with below.

\section{Polynomial Newton Systems}

The circumstances mentioned in the proof of the last theorem gives us the thought of trying to describe all polynomial Newton systems for which there are transversal commuting polynomial fields. The results turned out to be unexpected for us. The rest of the article is devoted to exposition of these results.

Consider a general polynomial Newton system, expanding its force function in the powers of the variable having the meaning of velocity:

$$
\begin{aligned}
& \dot{x}=-y, \\
& \dot{y}=q_{0}(x)+q_{1}(x) y+\ldots+q_{n}(x) y^{n} .
\end{aligned}
$$

The polynomials $q_{0}, \ldots, q_{n}$ in $x$ can have arbitrary degrees, but we assume that $q_{0}$ and $q_{1}$ have no constant terms and that the derivative $q_{0}$ at zero equals one. This means that the linear part of the system is the canonical oscillator.

As is well known, if a polynomial Newton system (8) is conservative, i.e., all $q_{k}$ with $k \geq 1$ vanish; then the oscillations described by the system are isochronous only in the trivial case of $q_{0}(x) \equiv x$. This fact was first established in [16], afterwards it was indicated in [1], and after many years it appeared in [17], where it received a new proof. Thus, in the conservative case, there arises no question of existence of a commuting system. Therefore, henceforth we assume that $n \geq 1$ and that the last polynomial $q_{n}$ does not vanish identically.

Theorem 5 If $n \geq 4$ then system (\$) cannot commute with any polynomial system nonproportional to it.

Proof. Suppose that a polynomial vector field $(r, s)$ commutes with the field $(-y, q)$, where $q$ is the right-hand side of the second equation in (8). As mentioned, the first relation in (4) allows us to express $s$ by means of (6). In this case the second relation in (幽) takes the form

$$
q_{x}\left(r-y r_{y}\right)+\left(y q_{y}-q\right) r_{x}+y^{2} r_{x x}-2 y q r_{x y}+q^{2} r_{y y}=0 .
$$

As in the proof of Theorem 4, this equality implies that the degree of the polynomial $r$ in the variable $y$ cannot exceed one, so that $r=r_{0}(x)+r_{1}(x) y$. Thus, the last summand

in (9) vanishes and leading is the coefficient of $y^{n+1}$ equal to $(n-3) q_{n} r_{1}^{\prime}$. Since $n>3$ and the polynomial $q_{n}$ is not equal to zero, we conclude that $r_{1}^{\prime}=0$ or $r_{1}=-c$, where $c$ is a constant.

Now, the maximal power of $y$ in (9) equals $n$ and the corresponding coefficient equals

$$
q_{n}^{\prime} r_{0}+(n-1) q_{n} r_{0}^{\prime}
$$


Its vanishing is possible only if $\left(q_{n} r_{0}^{n-1}\right)^{\prime}=0$; hence, $r_{0}$ too is a constant. It remains to observe that in these circumstances equation (9) is reduced to the form $q_{x} r_{0}=0$, where $q_{x}=1$ for $x=y=0$, which implies that $r_{0}=0$. Thus, $r=-c y$, and so $s=c q$, as it follows from (6).

Problem 3. Can a polynomial system of the form (8) with $n \geq 4$ be isochronous?

Anyway, such systems are absent from the list of the examples described in the articles known to us.

Theorem 6 If $n=2$ then a system of the form (8) commutes with no polynomial system nonproportional to it.

Proof. Here we use the same notations as in the proof of the preceding theorem. Observe that the highest power with which the variable $y$ enters in each summand of (9) for $n=2$ equals $m+2$, where $m$ is the degree of $r$ in $y$. Hence,

$$
(1-m) q_{2}^{\prime} r_{m}+(1-2 m) q_{2} r_{m}^{\prime}+r_{m}^{\prime \prime}+m(m-1) q_{2}^{2} r_{m}=0
$$

with $r_{m}$ the coefficient of $y^{m}$ in the expansion of $r$, representing a polynomial in $x$. Since $q_{2}$ is not identically zero, the last equality implies that $m$ is at most one. Certainly, it suffices to settle the case of $m=1$. Putting as before $r=r_{0}(x)+r_{1}(x) y$, we note that the equation is then transformed into $r_{1}^{\prime \prime}=q_{2} r_{1}^{\prime}$, which can hold only for a constant polynomial $r_{1}$.

Collecting the coefficients of $y^{2}$ in (9), we now arrive at the relation $r_{0}^{\prime \prime}=-\left(q_{2} r_{0}\right)^{\prime}$ which means that $r_{0}$ too is a constant. The further arguments are reduced to repeating what was said in the proof of the previous theorem: $r_{0}=0$ and the vector field $(r, s)$ is proportional to the field $(-y, q)$ of the system under study.

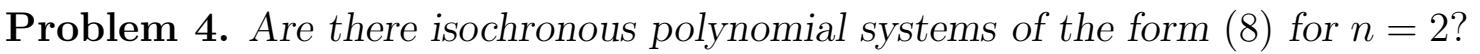

We hope that answers to the questions posed in this section will be related to a rather deep penetration into the isochronicity nature of polynomial systems. But already now it should be emphasized that the very statement of these questions became possible due to the remarkable idea that had combined concepts that, as it would seem, have a little in common: isochronicity of oscillations and commutation of vector fields.

\section{Isochronous Liénard Systems}

Of the two remaining classes of polynomial Newton systems, we first discuss one for which $n=1$. In this case the force function depends linearly on velocity and the system, known under the name of a Liénard system, acquires an especially attractive simple form:

$$
\begin{aligned}
& \dot{x}=-y, \\
& \dot{y}=q_{0}(x)+q_{1}(x) y .
\end{aligned}
$$

We recall that the linear parts of the systems under study represent the standard harmonic oscillator. 
Here we digress slightly from the main theme of our investigation, since in our opinion the question of commuting fields for systems of the form (10) is not very interesting for the following reasons: In [18] Sabatini studied Liénard systems with odd analytical coefficients $q_{0}$ and $q_{1}$ and obtained an exhaustive solution of the isochronicity problem in this case. Namely, he established that under the indicated conditions the origin is an isochronous center if and only if

$$
q_{0}(x)=x+\frac{I^{2}(x)}{x^{3}}, \quad \text { where } \quad I(x)=\int_{0}^{x} s q_{1}(s) d s .
$$

Furthermore, there are rather solid grounds to believe that, as far as the polynomial Liénard systems are concerned, there are no other cases of isochronicity, although nobody can prove this yet (in this connection, see the survey [14] and the references therein). Anyway, we are firmly convinced that, among all polynomial Liénard systems, only those belonging to the Sabatini class can commute with some transversal polynomial systems; furthermore, they comprise a small part even in this class. Clearly, in such situation, the question of commutation looses actuality, unless its study finds the end in itself.

We now formulate two problems whose positive solution would clearly speak in favor of the above-mentioned conjecture. Assume as before that $q_{0}$ and $q_{1}$ are polynomials. We arrange their primitives:

$$
Q_{0}(x)=\int_{0}^{x} q_{0}(s) d s, \quad Q_{1}(x)=\int_{0}^{x} q_{1}(s) d s .
$$

Problem 5. Is it true that the origin is a center of system (10) only in the following two cases: either $Q_{0}$ and $Q_{1}$ are even polynomials or $Q_{1}$ is a polynomial of $Q_{0}$ ?

This problem is cast by a beautiful result by Cherkas that he obtained in [19]. His theorem, on the one hand, guarantees the presence of a center under each of the above conditions and, on the other hand, enables us to reduce the problem to the following question: is it true that if a polynomial of one variable is represented as the sum of a power series in another polynomial which starts with a square of the variable and involves at least one odd power of this variable then the series terminates somewhere?

Problem 6. Is it true that if $Q_{1}$ is a polynomial of $Q_{0}$ then system (10) cannot be isochronous?

As an illustration of the second question, we consider the simplest class of systems satisfying the indicated condition:

$$
\begin{aligned}
& \dot{x}=-y, \\
& \dot{y}=f(x)(1+y),
\end{aligned}
$$

where $f(x)$ is a polynomial starting with $x$. We prove that the origin is always a center of the system, but it is never isochronous. 


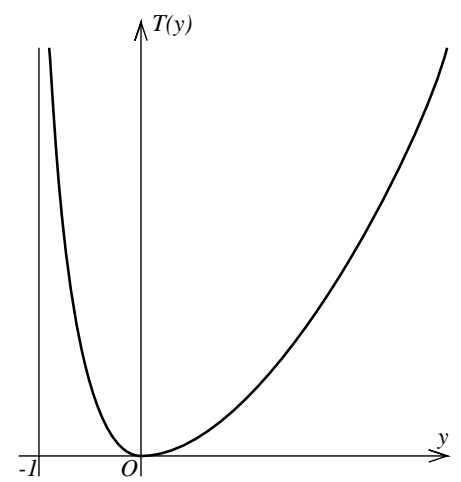

Fig. 4
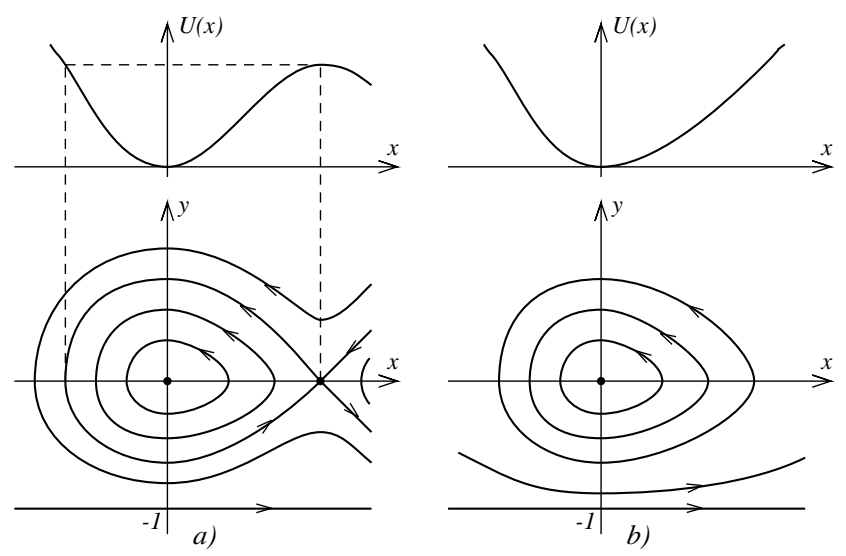

Fig. 5

First of all, by setting

$$
U(x)=\int_{0}^{x} f(s) d s, \quad T(y)=y-\ln (1+y),
$$

we find that the sum

$$
U(x)+T(y)=\frac{1}{2}\left(x^{2}+y^{2}\right)+\ldots
$$

serves as a first integral of our system. Next, we should use the fact that the function $T(y)$, defined for $y>-1$, has two intervals of strict monotonicity (Fig. 4) on each of which it takes all values from 0 to $+\infty$, and examine two possible cases: whether or not the system has stationary points other than zero.

In the first case, until $E>0$ attains the value of the polynomial $U$ at the critical point of $U$ nearest to zero (Fig. 5,a), the equation $U(x)+T(y)=E$ determines two functions $y$ of $x$ whose graphs form together a cycle of the system. This means that the singular point nearest to the origin lies on the boundary of the basin of the center and so this center cannot be isochronous. If there are no stationary points other than zero, then for the same reasons the basin of the center (Fig. 5,b) adheres immediately to the line $y=-1$ representing an orbit of the system along which the motion has unit velocity and, consequently, infinite duration, so that a long time is needed for traversing the nearby cycles.

The assumption that $f(x)$ is a polynomial is needed only for the primitive $U(x)$ to have two branches going to infinity in the case of the absence of nonzero roots and also for the period of a cycle around a center to depend analytically on the cycle.

\section{Centralizers of the Abel Systems}

We now consider the polynomial Abel systems, more precisely systems of the form (\$) with $n=3$, slightly changing their record:

$$
\begin{aligned}
& \dot{x}=-y, \\
& \dot{y}=q_{0}(x)+3 q_{1}(x) y+q_{2}(x) y^{2}+q_{3}(x) y^{3} .
\end{aligned}
$$

We do not know all isochronous Abel systems, but we can give a full description for those of them for which there are transversal commuting polynomial fields. 
Theorem 7 The field of system (11) commutes with some transversal polynomial vector field if and only if the following two conditions are satisfied:

$$
\begin{aligned}
& q_{2} q_{0}=3 q_{1}^{2}-q_{0}^{\prime}+1 \\
& q_{3} q_{0}^{2}=q_{1}^{3}-q_{1}^{\prime} q_{0}+q_{1} .
\end{aligned}
$$

Proof. Let $(r, s)$ be a polynomial field commuting with the field $(-y, q)$ of system (11). Then $s$, as was constantly mentioned, is expressed by formula (6) and $r$ is found from equation (9). Repeating word for word the arguments presented at the analogous place in the proof of Theorem 4, incidentally, also devoted to one of the equations in the Abel class, we conclude that in this case as well we have $r=r_{0}+r_{1} y$, where $r_{0}$ and $r_{1}$ are polynomials of $x$.

Elementary calculations of the coefficients of various powers of $y$ in (9) lead us to four relations, the first two of which are as follows:

$$
q_{0}^{\prime} r_{0}-q_{0} r_{0}^{\prime}=0, \quad q_{1}^{\prime} r_{0}-q_{0} r_{1}^{\prime}=0
$$

The general solution of these equation have the form

$$
r_{0}=c_{0} q_{0}, \quad r_{1}=c_{0} q_{1}+c_{1},
$$

where $c_{0}$ and $c_{1}$ are arbitrary constants. If $c_{0}=0$ then it is easy to see that the commuting field is proportional to the original field. Therefore, henceforth we may assume that $c_{0}=1$ and $c_{1}=0$.

Now, if we write down the third of the above-mentioned four relations,

$$
q_{2}^{\prime} r_{0}+q_{2} r_{0}^{\prime}+r_{0}^{\prime \prime}-6 q_{1} r_{1}^{\prime}=0
$$

by using the equalities $r_{0}=q_{0}$ and $r_{1}=q_{1}$, we obtain the equation

$$
q_{2}^{\prime} q_{0}+q_{2} q_{0}^{\prime}+q_{0}^{\prime \prime}-6 q_{1} q_{1}^{\prime}=\left(q_{2} q_{0}\right)^{\prime}+\left(q_{0}^{\prime}\right)^{\prime}-3\left(q_{1}^{2}\right)^{\prime}=0
$$

which is easy to solve and which leads to the formula

$$
q_{2} q_{0}+q_{0}^{\prime}-3 q_{1}^{2}=c_{2}
$$

where $c_{2}$ is a constant. Factually, $c_{2}=1$, since, as we have agreed, $q_{0}(0)=q_{1}(0)=0$ and $q_{0}^{\prime}(0)=1$. This proves the necessity of the first condition in (12).

To prove the second condition, we use the last relation which ensues from (9):

$$
q_{3}^{\prime} r_{0}+2 q_{3} r_{0}^{\prime}+r_{1}^{\prime \prime}-q_{2} r_{1}^{\prime}=0
$$

Expressing $r_{0}, r_{1}$ and $q_{2}$ in terms of $q_{0}$ and $q_{1}$, we obtain the differential equation

$$
q_{3}^{\prime} q_{0}^{2}+2 q_{3} q_{0} q_{0}^{\prime}+q_{0} q_{1}^{\prime \prime}+q_{0}^{\prime} q_{1}^{\prime}-3 q_{1}^{2} q_{1}^{\prime}-q_{1}^{\prime}=0
$$


which integrates without effort:

$$
q_{3} q_{0}^{2}+q_{0} q_{1}^{\prime}-q_{1}^{3}-q_{1}=c_{3}
$$

Here $c_{3}$ is one more constant. But now it relates to an expression vanishing at $x=0$, so that $c_{3}=0$, and the necessity of the second condition in (12) is established as well.

Sufficiency of (12) for existence of a sought commuting system follows from the obvious conversability of our logical constructions. Also, it can be easily verified by inserting the found $r$ in (9).

As we see from the proof of the theorem, in the case under consideration there is exactly one sought commuting field $(r, s)$ which is, up to a constant factor and a trivial summand, proportional to the original Abel field $(-y, q)$ :

$$
r=q_{0}+q_{1} y, \quad s=y r_{x}-q r_{y}
$$

Straightforward application of Theorem 7 to construction of commuting polynomial systems encounters certain difficulties: if we choose the polynomials $q_{0}$ and $q_{1}$ at random then the functions $q_{2}$ and $q_{3}$ calculated in accordance with formulas (12) turn out as a rule merely rational. Therefore, it is desirable to have an explicit description for all polynomial Abel systems whose coefficients are bound by relations (12). This problem turned out to be solvable.

Theorem 8 A polynomial Abel system (11) commutes with some transversal polynomial system if and only if it has the following form:

$$
\begin{aligned}
& \dot{x}=-y, \\
& \dot{y}=\left(x+a^{2} x^{3}\right)(1+h(x) y)^{3}+3 a x y(1+h(x) y)^{2}-h^{\prime}(x) y^{3},
\end{aligned}
$$

where $a$ is an arbitrary number and $h(x)$ is an arbitrary polynomial.

It is easy to see that the coefficient of $y^{3}$ in (14) vanishes only if $h(x) \equiv 0$ in which case the system transforms into the isochronous Kukles system. Even in the simplest case in which $a=0$ and $h(x) \equiv 1$, we obtain a new example of an isochronous Abel system:

$$
\begin{aligned}
& \dot{x}=-y, \\
& \dot{y}=x(1+y)^{3} .
\end{aligned}
$$

In this case it is obvious that the origin is a center. Isochronicity of the center is guaranteed by the membership of the system in the class (14), although here this fact can be verified by straightforward calculations.

We turn to proving Theorem 8. In all forthcoming lemmas, the talk is about the polynomial coefficients of the second equation of the Abel system (11) which satisfy relations (12) of Theorem 7 .

Lemma 1 The polynomial $q_{0}$ has the form $q_{0}(x)=x+A x^{3}$, where $A$ is a constant. 
Proof. The second condition in (12) shows that the polynomial $q_{1}\left(1+q_{1}^{2}\right)$ is divisible by $q_{0}$. Then it is easy to see that $q_{0}$ admits the decomposition $q_{0}=p_{1} p_{2}$ into the product of the polynomials

$$
p_{1}(x)=x+O\left(x^{2}\right) \quad \text { and } \quad \mathrm{p}_{2}(\mathrm{x})=1+\mathrm{O}(\mathrm{x})
$$

which divide $q_{1}$ and $1+q_{1}^{2}$ respectively. Since

$$
q_{2} q_{0}=3 q_{1}^{2}-q_{0}^{\prime}+1=3\left(q_{1}^{2}+1\right)-q_{0}^{\prime}-2,
$$

the difference $q_{0}^{\prime}-1$ is divisible by $p_{1}$ and the sum $q_{0}^{\prime}+2$, by $p_{2}$. Using the equality $q_{0}^{\prime}=p_{1}^{\prime} p_{2}+p_{1} p_{2}^{\prime}$, we arrive at the following important conclusion: there are polynomials $h_{1}$ and $h_{2}$ such that

$$
p_{1}^{\prime} p_{2}-1=h_{1} p_{1}, \quad p_{1} p_{2}^{\prime}+2=h_{2} p_{2} .
$$

Before going on further, we exclude one particular case. Namely, as the first equality in (15) shows, if $p_{2} \equiv 1$ then $p_{1}^{\prime}-1=h_{1} p_{1}$, which is possible only for $p_{1} \equiv x$; whence, $q_{0} \equiv x$. This is in a full agreement of the claim of the lemma, if we take $A=0$. Thus, the degree of $p_{2}$ can be assumed positive. But then, as follows from (15), the degrees of $h_{1}$ and $h_{2}$ are less exactly by one than those of $p_{2}$ and $p_{1}$.

We now multiply the first relation in (15) by $h_{2}$ and express the resultant product $h_{2} p_{2}$ by using the second relation. Interchanging the words "one" and "two" in the last proposition, we perform an analogous procedure again. At a result, we obtain two interesting equalities:

$$
2 p_{1}^{\prime}-h_{2}=p_{1}\left(h_{1} h_{2}-p_{1}^{\prime} p_{2}^{\prime}\right), \quad-p_{2}^{\prime}+2 h_{1}=p_{2}\left(h_{1} h_{2}-p_{1}^{\prime} p_{2}^{\prime}\right) .
$$

They are interesting, because they make their left-hand sides divisible by polynomials of higher degrees and so yield the formulas

$$
2 h_{1}=p_{2}^{\prime}, \quad h_{2}=2 p_{1}^{\prime} .
$$

In this case, both relations of (15) become a sole equality:

$$
2 p_{1}^{\prime} p_{2}-p_{1} p_{2}^{\prime}=2
$$

It is from the last relation that we derive the necessary conclusions.

First of all, for the mutual compensation of the coefficients of the highest powers of the variable on the left-hand side of (16), it is necessary that the degree of $p_{2}$, being nonzero, must be twice as large than the degree of $p_{1}$. In that event, we can choose a factor $A$ so that the degree of $p_{3}=p_{2}-A p_{1}^{2}$ be strictly less than that of $p_{2}$. However, equality (16) remains valid if we substitute $p_{3}$ for $p_{2}$ in it:

$$
2 p_{1}^{\prime} p_{3}-p_{1} p_{3}^{\prime}=2
$$

These arguments about degrees now lead us to another conclusion: $p_{3} \equiv c$, where $c$ is a constant. Thus, $p_{2}=A p_{1}^{2}+c$, and (16) is extremely simplified: $c p_{1}^{\prime}=1$. Involving the initial data, whence we infer that $c=1$. Therefore, $p_{1} \equiv x$ and $p_{2} \equiv A x^{2}+1$, which completes the proof of the lemma. 
Lemma 2 There must exist a real number a and a polynomial $h(x)$ such that $A=a^{2}$, where $A$ is the coefficient mentioned in the preceding lemma and $q_{1}$ has the form

$$
q_{1}(x)=a x+\left(x+a^{2} x^{3}\right) h(x) .
$$

Proof. In view of Lemma 1, the first relation in (12) looks like

$$
q_{2}\left(x+A x^{3}\right)=3\left(q_{1}^{2}-A x^{2}\right) .
$$

Were the coefficient $A$ negative, the left-hand side of the equality would have nonzero roots, whereas the right-hand side would not. This proves the possibility of writing $A=a^{2}$. Then the preceding relation can be written otherwise:

$$
q_{2} x\left(1+a^{2} x^{2}\right)=3\left(q_{1}-a x\right)\left(q_{1}+a x\right) .
$$

It is clear that one of the nonconstant factors on the right-hand side must be divisible by the irreducible polynomial $1+a^{2} x^{2}$. Since the sign of the number $a$ was not fixed, we may assume that this is the first factor. Moreover, the chosen factor is also divisible by $x$. Thus, the function $h(x)$, necessarily defined as the quotient of $q_{1}(x)-a x$ by $x+a^{2} x^{3}$, is a polynomial.

The next lemma, whose proof is reduced to simple calculations, is stated only for the reader's convenience.

Lemma 3 If $q_{0}$ and $q_{1}$ are expressed in terms of $a$ and $h$ as it is said in the previous two lemmas, then equations (12) is uniquely solvable for $q_{2}$ and $q_{3}$ in the class of polynomials and their solutions have the form

$$
\begin{aligned}
& q_{2}(x)=6 a x h(x)+\left(x+a^{2} x^{3}\right) h^{2}(x), \\
& q_{3}(x)=3 a x h^{2}(x)+\left(x+a^{2} x^{3}\right) h^{3}(x)-h^{\prime}(x) .
\end{aligned}
$$

To complete the proof of Theorem 8, it now remains only a little: we have to insert the expressions for coefficients which are indicated in the three lemmas into the second equation of (11) and verify that the result is system (14). Also we should observe that these coefficients satisfy relations (12), because it is from exactly these relations that the last two of them were found.

\section{Conditions for a Center in Smooth Abel Systems}

Describing polynomial Abel systems commuting with transversal polynomial fields, we supposed nothing about their behavior near the origin in advance. Meanwhile, if the linear part of a system is a harmonic oscillator then the origin can be not only a center but also a focus. In the latter case, it is quite possible that there exists a nontrivial commuting field, in which case we also observe some kind of isochronicity, namely: a point moving along a spiral around a focus returns to an arbitrarily given radial orbit of the commuting system by the same time.

Thus, we have to clarify which of the found Abel systems have a center at the origin. Furthermore, it is interesting to understand the meaning of the enigmatic form of these systems which is indicated in Theorem 8. Answers to these questions are given in the following theorem which holds not only for polynomial systems. 
Theorem 9 For an arbitrary number $a$ and an arbitrary smooth function $h(x)$, the system of the form (14) in the variables

$$
X=x, \quad Y=\frac{y}{1+h(x) y}
$$

is collinear to the isochronous Kukles system:

$$
\begin{aligned}
& \lambda \dot{X}=-Y \\
& \lambda \dot{Y}=X+3 a X Y+a^{2} X^{3}
\end{aligned}
$$

where $\lambda=1-h(X) Y$. In particular, if $a=0$ then the system is simply reduced to the harmonic oscillator. Moreover, the origin is always an isochronous center of system (14).

Proof. Verification of reducibility of one system to another when a change of variables is given explicitly requires nothing but accurate calculations. Collinearity of a system to the Kukles system, although with a variable factor, shows that the origin is a center. Isochronicity of the center follows from the fact that the field of system (14) commutes with the transversal field $(r, s)$ if we define the latter by formulas (13). This is easy to check by direct calculation of the Poisson bracket or, equivalently, by verification of equation (9).

The just proven theorem can be seen as an assertion reflecting a curious property of the isochronous Kukles system. Indeed, if we multiply or divide the field of this system by an arbitrary function not taking zero values in a neighborhood of the center then the orbits of the new system are certainly the same but the law of motion along them changes. However, as we see, if this field is divided by a function of the form $1-h(X) Y$, where $h(X)$ can be arbitrary, then the total time of circuit around the center remains the same and isochronicity is preserved. In our opinion, this extraordinary phenomenon which, judging by all appearances, should also be encountered in other systems deserves an independent study. To state it more precisely, it would be interesting to clarify the changes of the velocity of the motion along orbits of an isochronous system which do not influence the period of cycles.

Formulas (12) reduce system (11) to the form (14) only in the case of polynomial vector fields. If we remove this restriction and consider the Abel systems with arbitrary smooth coefficients then conditions (12) distinguish an essentially wider class of systems. It turns out that in the general case as well these conditions not only guarantee existence of a transversal commuting field but also ensure the presence of a center at the origin.

Theorem 10 For arbitrary smooth functions $q_{0}(x)=x+O\left(x^{2}\right)$ and $q_{1}(x)=O(x)$, relations (12) uniquely determine smooth functions $q_{2}(x)$ and $q_{3}(x)$ which are also analytical if so are $q_{0}(x)$ and $q_{1}(x)$. Moreover, each Abel system (11) with these coefficients has an isochronous center at the origin.

Proof. Since the order of $q_{0}(x)$ in $x$ is exactly one; to prove solvability of equations (12) in the class of smooth or analytical functions, it suffices to observe that the orders of their right-hand sides are at least 1 and 2 respectively. 
It is curious to notice that systems (11) with coefficients connected by relations (12) are contained in the class of integrable Abel systems which was recently found by Lukashevich and described by him in [20]. Following his constructions, we can obtain a first integral of our system which turns out to be equal to the sum of the squares of two functions $X$ and $Y$ of the variables $x$ and $y$. Omitting the details of intermediate calculations, we only state the result. Namely, we define these functions by the formulas

$$
X=x e^{I(x)}, \quad Y=\frac{x y e^{I(x)}}{q_{0}(x)+q_{1}(x) y},
$$

where $I(x)$ stands for the integral

$$
I(x)=\int_{0}^{x} \frac{u-q_{0}(u)}{u q_{0}(u)} d u .
$$

Since the integrand clearly has no singularities at zero, the integral $I(x)$ represents a smooth function of $x$. As it is easy to calculate,

$$
\frac{\partial(X, Y)}{\partial(x, y)}=1
$$

at the point $x=y=0$, so that the transformation $x \rightarrow X, y \rightarrow Y$ is invertible on some neighborhood of the origin. Moreover, system (11), with the coefficients $q_{2}$ and $q_{3}$ expressed in terms of $q_{0}$ and $q_{1}$ by means of (12), in the new variables is reduced to the form

$$
\mu \dot{X}=-Y, \quad \mu \dot{Y}=X,
$$

where the variable factor $\mu$ equals 1 at $X=Y=0$. Thus, the origin is a center of the system under consideration.

As far as isochronicity is concerned, here it suffices to repeat what was said in this connection in the proof of the previous theorem: as it is easy to check, the field of the system commutes with the field $(r, s)$ transversal to it in a neighborhood of the origin and defined by relations (13).

However, in this case isochronicity of the center follows also from other arguments. Namely, the above-mentioned factor $\mu$ in the new variables $X$ and $Y$ has the form $1-f(X) Y$, where $f(X)$ is the ratio $q_{1}(x) / X$ expressed in terms of $X$, and it is easy to verify by passage to polar coordinates that such a factor does not change the period of cycles of the harmonic oscillator.

Closing the article, we return to the polynomial Abel systems and sum up some conclusions. First, as Theorem 7 and, especially, Theorem 8 demonstrate, such systems commute with transversal polynomial fields only in exceptional cases. Second, the very fact of commutation, as Theorem 9 or more general Theorem 10 asserts, guarantees the presence of a center at the origin and this center must be isochronous. Third, system of the form (5) and Theorems 3 and 4 devoted to them recall us that Theorem 8 describes far from all isochronous polynomial Abel systems.

Problem 7. Find all polynomial Abel systems possessing isochronous centers.

Acknowledgement We are thankfull to S.Treskov and V.Vershinin for your help during the preparation of the article. 


\section{References}

[1] V. V. Amel'kin, N. A. Lukashevich, and A. P. Sadovskii, Nonlinear Oscillations in the Systems of Second Order [in Russian], Belorus. Univ., Minsk (1982).

[2] P. Mardešić, C. Rousseau, and B. Toni, Linearization of isochronous centers, J. Differential Equations, 121, No. 1, 67-108 (1995).

[3] P. Mardešić, L. Moser-Jauslin, and C. Rousseau, Darboux linearization and isochronous centers with a rational first integral, J. Differential Equations, 134, No. 2, 216-268 (1997).

[4] M. Villarini, Regularity properties of the period function near a center of a planar vector field, Nonlinear Anal., 19, No. 8, 787-803 (1992).

[5] N. A. Lukashevich, Isochronicity of a center for certain systems of differential equations, Dfferential Equations (translation from Differentsial'nye Uravneniya), 1, No. 5, 295-302 (1965).

[6] V. I. Arnol'd, Mathematical Methods of Classical Mechanics [in Russian], Nauka, Moscow (1974).

[7] M. Sabatini, Characterizing isochronous centres by Lie brackets, Differential Equations Dynam. Systems, 5, No. 1, 91-99 (1997).

[8] C. J. Christopher and J. Devlin, Isochronous centers in planar polynomial systems, SIAM J. Math. Anal., 28, No. 1, 162-177 (1997).

[9] M. Sabatini, Quadratic Isochronous Centres Commute [Preprint, 461], Trento (1995).

[10] Mathematica. Version 2.2, Champaign, Illinois (1994). (Wolfram Research, Inc.)

[11] E. Sáez and I. Szántó, One-parameter family of a cubic Kolmogorov system with an isochronous center, Collect. Math., 48, No. 3, 297-301 (1997).

[12] M. Sabatini, A Connection Between Isochronous Hamiltonian Centers and the Jacobian Conjecture [Preprint, No. 460], Trento (1995).

[13] J. Devlin, Coexisting isochronous and nonisochronous centres, Bull. London Math. Soc., 28, No. 134, 495-500 (1996).

[14] J. Chavarriga and M. Sabatini, A survey of isochronous centers, in: Winter School on Polynomial Vector Fields, 11-12 December, 1997, Lleida.

[15] I. I. Pleshkan, On isochronicity conditions of the system of two differential equations, Dfferential Equations (translation from Differentsial'nye Uravneniya), 4, No. 11, 19911993 (1968). 
[16] V. V. Amel'kin, To the question on isochronicity of a center for two-dimensional analytic differential systems, Dfferential Equations (translation from Differentsial'nye Uravneniya), 13, No. 6, 971-980 (1977).

[17] C. Chicone and M. Jacobs, Bifurcation of critical periods for plane vector fields, Trans. Amer. Math. Soc., 312, No. 2, 433-486 (1989).

[18] M. Sabatini, On the period function of Liénard systems, J. Differential Equations (to appear).

[19] L. A. Cherkas, On conditions of the center for some equations of the type $y y^{\prime}=P(x)+$ $Q(x) y+R(x) y^{2}$, Dfferential Equations (translation from Differentsial'nye Uravneniya), 8, No. 8, 1435-1439 (1972).

[20] N. A. Lukashevich, Special Abel equations, Dfferential Equations (translation from Differentsial'nye Uravneniya), 34, No. 2, 282-284 (1998).

Sobolev Institute of Mathematics, 630090, Novosibirsk, Russia. E-mail: volok@math.nsc.ru 